\title{
PSMA-ligand uptake can serve as a novel biomarker in primary prostate cancer to predict outcome after radical prostatectomy
}

Hui Wang ${ }^{1 *+}\left(\mathbb{0}\right.$, Thomas Amiel ${ }^{2}$, Christoph Würnschimmel ${ }^{3}$, Thomas Langbein ${ }^{1}$, Katja Steiger ${ }^{4}$, Isabel Rauscher ${ }^{1}$, Thomas Horn' ${ }^{2}$, Tobias Maurer ${ }^{3,5}$, Wolfgang Weber ${ }^{1}$, Hans-Juergen Wester ${ }^{6}$, Karina Knorr ${ }^{1 \dagger}$ and Matthias Eiber ${ }^{1+}$

\begin{abstract}
Background: The prostate-specific membrane antigen (PSMA) is a relevant target in prostate cancer, and immunohistochemistry studies showed associations with outcome. PSMA-ligand positron emission tomography (PET) is increasingly used for primary prostate cancer staging, and the molecular imaging TNM classification (miTNM) standardizes its reporting. We aimed to investigate the potential of PET-imaging to serve as a noninvasive imaging biomarker to predict disease outcome in primary prostate cancer after radical prostatectomy (RP).

Methods: In this retrospective analysis, 186 primary prostate cancer patients treated with RP who had undergone a ${ }^{68} \mathrm{Ga}$-PSMA-11 PET up to three months prior to the surgery were included. Maximum standardized uptake value $\left(S U V_{\text {max }}\right), S U V_{\text {mean }}$, tumor volume (TV) and total lesion (TL) were collected from PET-imaging. Moreover, clinicopathological information, including age, serum prostate-specific antigen (PSA) level, and pathological characteristics, was assessed for disease outcome prediction. A stage group system for PET-imaging findings based on the miTNM framework was developed.
\end{abstract}

Results: At a median follow-up after RP of 38 months (interquartile range (IQR) 22-53), biochemical recurrence $(B C R)$ was observed in 58 patients during the follow-up period. A significant association between a positive surgical margin and miN status (miN1 vs. miN0, odds ratio (OR): 5.428, $p=0.004$ ) was detected. miT status (miT $\geq 3 a$ vs. miT $<3$, OR: 2.696, $p=0.003$ ) was identified as an independent predictor for Gleason score (GS) $\geq 8$. Multivariate Cox regression analysis indicated that PSA level (hazard ratio (HR): 1.024, $p=0.014$ ), advanced GS (GS $\geq 8$ vs. GS $<8$, HR: 3.253, $p<0.001$ ) and miT status (miT $\geq 3$ a vs. miT $<3, H R: 1.941, p=0.035$ ) were independent predictors for BCR. For stage I disease as determined by PET-imaging, a shorter BCR-free survival was observed in the patients with higher SUV max $_{\text {(IA }}$ vs. IB stage, log-rank, $p=0.022$ ).

Conclusion: Preoperative miTNM classification from ${ }^{68} \mathrm{Ga}$-PSMA-11 PET correlates with postoperative GS, surgical margin status and time to BCR. The association between miTNM staging and outcome proposes ${ }^{68} \mathrm{Ga}-\mathrm{PSMA}-11 \mathrm{PET}$ as a novel non-invasive imaging biomarker and potentially serves for ancillary pre-treatment stratification.

Keywords: Biochemical recurrence, ${ }^{68} \mathrm{Ga}-\mathrm{PSMA}-11$ PET, miTNM classification, Prostate cancer

*Correspondence: huihui.wang@tum.de

${ }^{\dagger}$ Hui Wang, Karina Knorr and Matthias Eiber: Joint senior authorship

${ }^{1}$ Department of Nuclear Medicine, Klinikum rechts der Isar, Technical

University Munich, Ismaninger Str. 22, 81675 Munich, Germany

Full list of author information is available at the end of the article

\section{Background}

Approximately $30 \%$ to $40 \%$ of prostate cancer patients will fail primary treatment requiring further disease management $[1,2]$. Traditional risk factors, including preoperative serum prostate-specific antigen (PSA) level [3-6], pathological stage [7] and Gleason score (GS) [5-9], are 
widely used for biochemical recurrence (BCR) prediction. However, there is growing interest in identifying novel biomarkers to improve BCR prediction accuracy of prostate cancer patients after radical prostatectomy (RP).

In the last few years positron emission tomography (PET) probes targeting prostate-specific membrane antigen (PSMA) has significantly improved detection and localization of disease in primary and recurrent prostate cancer [1, 10-12]. PSMA is a type II integral membrane glycoprotein with folate hydrolase activity, internalization after activation and is encoded by the FOLH1 gene [13, 14]. PSMA expression increases progressively in highergrade prostate tumor cells and metastatic lesions $[15,16]$.

Increased PSMA expression in immunohistochemistry was more often observed in pathological stage III or IV tumors $(51 \%)$ compared to stage I and II tumors (32\%, $p=0.029$ ) [17]. High-level PSMA expression in immunohistochemistry was associated with a higher risk of BCR and overall survival in several studies [16, 17]. Finally, expression of membranous PSMA is also associated with higher rates of defective deoxyribonucleic acid (DNA) damage repair gene [18]

In the last two decades, for different tumor entities results from imaging were introduced as non-invasive quantitative biomarkers. For fluorodeoxyglucose (FDG)PET-imaging, Wieder et al. have demonstrated that mean standardized uptake value $\left(\mathrm{SUV}_{\text {mean }}\right)$ can be used to preoperatively predict histopathological response in esophageal squamous cell carcinoma (ESCC) patients. A decrease in $\mathrm{SUV}_{\text {mean }}$ of $44 \% \pm 15 \%$ from responders and $21 \% \pm 14 \%$ from non-responders $(p=0.0055)$ was observed after radiochemotherapy [19]. Besides, Giovacchini et al. have observed that the positive results of ${ }^{11} \mathrm{C}$-choline PET/computed tomography (CT) predict prostate cancer-specific survival in patients after RP [20]. For metastatic castration-resistant prostate cancer (mCRPC), the bone scan index (BSI) [21] and quantitative parameter from PET have been reported to serve as potential predictive biomarkers for bone tumor burden $[22,23]$.

To standardize reporting of PSMA-targeted PETimaging, a unified molecular-imaging TNM classification (miTNM, version 1.0) has been recently introduced [24]. It is envisioned that its system classifying tumor extent similar to the pathological TNM-system might serve as qualitative imaging biomarker potentially stratifying disease outcome.

The aim of our retrospective analysis was to investigate the potential of quantitative and qualitative parameters from ${ }^{68} \mathrm{Ga}$-PSMA-11 PET to serve as non-invasive imaging biomarkers to predict $\mathrm{BCR}$ in primary prostate cancer after RP allowing for ancillary preoperative risk stratification.

\section{Methods}

Patient selection

We screened the institutions' database for all patients who underwent ${ }^{68} \mathrm{Ga}$-PSMA-11 PET imaging maximum 3 months prior the RP between January 2013 and August 2017. All patients who received neoadjuvant therapy prior to RP had PSA-persistence after RP or in whom follow-up data were missing were excluded. Finally, 186 patients with D'Amico intermediate- to high-risk primary prostate cancer were included in this retrospective study (Additional file 1: Fig. S1). Table 1 summarizes the clinical and histopathological characteristics. BCR was defined as a serum PSA level rising above $0.2 \mathrm{ng} / \mathrm{ml}$. The primary endpoint was time to BCR. The time to BCR was calculated from the date of surgery. The retrospective analysis has been approved by the Ethics Committee of the Technical University Munich (750/20 S-KH).

Table 1 Patient characteristics

\begin{tabular}{|c|c|}
\hline Characteristic & Patients \\
\hline Age (years), median (IQR), $n=186$ & $68(61-72)$ \\
\hline iPSA (ng/ml), median (IQR), $n=184^{\mathrm{a}}$ & $9.7(6.5-15.1)$ \\
\hline $\begin{array}{l}\text { Administered }{ }^{68} \mathrm{Ga} \text {-PSMA-11 activity (MBq), median (IQR), } \\
n=185^{\mathrm{b}}\end{array}$ & $139(112-156)$ \\
\hline Time PET to RP (day), median (IQR), $n=186$ & $26(13-46)$ \\
\hline \multicolumn{2}{|l|}{ Gleason score in surgical specimen, no. (\%), $n=186$} \\
\hline 6 & $11(5.9 \%)$ \\
\hline $7 a$ & $63(33.9 \%)$ \\
\hline $7 b$ & $59(31.7 \%)$ \\
\hline 8 & $28(15.1 \%)$ \\
\hline 9 & $25(13.4 \%)$ \\
\hline \multicolumn{2}{|l|}{ Pathological stage, no. (\%), $n=186$} \\
\hline \multicolumn{2}{|l|}{ pT status } \\
\hline $2 \mathrm{a}$ & $11(5.9 \%)$ \\
\hline $2 b$ & $10(5.4 \%)$ \\
\hline $2 c$ & $71(38.2 \%)$ \\
\hline $3 a$ & $49(26.3 \%)$ \\
\hline $3 b$ & $44(23.7 \%)$ \\
\hline 4 & $1(0.5 \%)$ \\
\hline \multicolumn{2}{|l|}{ pN status } \\
\hline 0 & $154(82.8 \%)$ \\
\hline 1 & $32(17.2 \%)$ \\
\hline \multicolumn{2}{|l|}{ Surgical margin, no. (\%), $n=180^{\circ}$} \\
\hline Negative & 152 (84.4\%) \\
\hline Positive & $28(15.6 \%)$ \\
\hline
\end{tabular}

IPSA initial PSA, IQR interquartile range, PET positron emission tomography, PSA prostate-specific antigen, $R P$ radical prostatectomy

${ }^{a}$ iPSA of two patients were unavailable

${ }^{b}$ The injected dose of ${ }^{68} \mathrm{Ga}-\mathrm{PSMA}-11$ from one patient was unavailable

${ }^{\mathrm{C}}$ The status of surgical margin from six patients were unavailable 


\section{Imaging protocol}

The synthesis of ${ }^{68}$ Ga-PSMA-11 [25] was described previously [26]. Patients were intravenously injected with a median of $139 \mathrm{MBq}$ of ${ }^{68} \mathrm{Ga}$-PSMA-11 (interquartile range (IQR) 112-156). PET acquisition was started at a median of 54 min (IQR 49-65) after the tracer injection. Nighty-three patients underwent ${ }^{68} \mathrm{Ga}$-PSMA-11 $\mathrm{PET} / \mathrm{CT}$ using a Biograph $\mathrm{mCT}$ flow scanner (Siemens Medical Solutions, Erlangen, Germany), and 93 patients underwent ${ }^{68} \mathrm{Ga}$-PSMA-11 PET/magnetic resonance imaging (MRI) using an integrated whole-body PET/MRI system (Siemens Biograph mMR, Erlangen, Germany). Details on PET/CT and PET/MRI acquisition were described previously $[27,28]$.

\section{Imaging analysis}

${ }^{68} \mathrm{Ga}-\mathrm{PSMA}-11$ PET/CT and ${ }^{68} \mathrm{Ga}-\mathrm{PSMA}-11$ PET/ MRI images were evaluated by one nuclear medicine physician blinded to the postoperative histopathological results. All lesions were reannotated by two experienced board-certified nuclear medicine physicians. Any focal or diffuse tracer uptake in the prostate or extra-prostatic lesions above the surrounding background and not associated with physiological uptake was considered suspicious for malignancy. One circular region in transaxial slices was drawn over the prostate and over every extra-prostatic lesion automatically adapted to a three-dimensional volume of interest (VOI) using Syngo.Via (Siemens Healthineers, Erlangen, Germany). A $40 \%$ isocontour of the SUVmax was used to determine the SUVmean. Typical pitfalls in PSMA-ligand PET including low to moderate PSMA uptake correlated with osteoblastic changes (i.e., fractures or degenerative changes), celiac, and ganglia were taken into consideration [29]. $\mathrm{SUV}_{\max }, \mathrm{SUV}_{\text {mean }}$, tumor volume (TV), and total lesion (TL) of every VOI were calculated. Prostatic and extra-prostatic lesions were classified according to miTNM classification [24]. In PSMA-PET negative primary tumors, the CT or MRI part of the hybrid PET-exam was used for miTNM stage group determination.

Similar to the structure of the American Joint Committee on Cancer (AJCC) Prostate Cancer Prognostic Stage Groups [30], we established a stage group system using the different grades from the miTNM staging system (Table 2). To allow the discrimination into different risk groups based on the characteristics of the primary tumor, we used a SUV ${ }_{\max }$ cut-off of 5.4 to subgroup stage I disease into stage IA and IB. The cut-off was derived from a recent study proposing it the optimal cut-off to distinguish between GS $\leq 7 \mathrm{a}$ and $\mathrm{GS} \geq 7 \mathrm{~b}$ [31].
Table 2 Proposed miTNM stage groups for ${ }^{68} \mathrm{Ga}$-PSMA-11 PET

\begin{tabular}{lllll}
\hline Stage Group & miT & miN & miM & SUV $_{\text {max }}$ \\
\hline IA & 2 & 0 & 0 & $<5.4$ \\
IB & 2 & 0 & 0 & $\geq 5.4$ \\
IIA & 3 & 0 & 0 & Any \\
IIB & 4 & 0 & 0 & Any \\
III & Any & 1 and 2 & 0 & Any \\
IV & Any & Any & 1 & Any \\
\hline
\end{tabular}

PET positron emission tomography, PSMA prostate-specific membrane antigen, SUV standardized uptake value

\section{Statistical analysis}

Descriptive statistics were used to display continuous variables as the median and IQR with 25th and 75th percentiles (Q1-Q3), mean \pm standard deviation (SD), as well as percentages. The association between pathological results and ${ }^{68} \mathrm{Ga}$-PSMA-11 PET findings was investigated with uni- and multivariate Logic regression analyses, and the corresponding odds ratios (OR) and $95 \%$ confidence intervals (CI) were calculated. Postoperative BCR-free survival was estimated using the Kaplan-Meier method and compared between groups using the Log-rank test. Moreover, uni- and multivariable Cox regression analysis were performed to determine the ability of clinicopathological factors and ${ }^{68} \mathrm{Ga}$-PSMA-11 PET findings to predict BRC after RP, and the corresponding hazard ratios (HR) and 95\% CI were calculated. The multivariable model only included parameters with a significant association on univariable analysis. A $p$ value of 0.05 was used as the cut-off for statistical significance.

Given its low sample size $(n=7)$ the miM1 subgroup was excluded for univariable and multivariable analysis.

Statistical evaluation was performed with IBM SPSS Statistics Version 20 (Armonk, NY, USA), and the figures were generated using GraphPad Prism Version 8 (San Diego, California, USA).

\section{Results}

\section{Histopathological patient characteristics}

On post-operative histopathology, a total of 133 (71.5\%) patients had a GS $<8$, and $53(28.5 \%)$ of the patients had a GS 8 or 9. Lymph node metastases were detected in $32(17.2 \%)$ patients. pT3a, pT3b and pT4 disease was present in $49(26.3 \%), 44(23.7 \%)$ and $1(0.5 \%)$ patient, respectively. Twenty-eight (15.6\%) patients had positive surgical margins (R1) (Table 1). At a median follow-up of 38 months (IQR 22-53), BCR was observed in 58 (31.2\%) patients during the follow-up period. 
${ }^{68} \mathrm{Ga}-\mathrm{PSMA}-11$ PET findings miTNM staging and miTNM stage groups

In $67.2 \%(n=125)$ of patients, the primary tumor was classified as miT2, 90.3\% $(n=168)$ were classified as miN0, 3.8\% $(n=7)$ were classified as miN1, 5.9\% $(n=11)$ were classified as $\mathrm{miN} 2$, and $96.2 \%(n=179)$ were classified as miM0. ${ }^{68}$ Ga-PSMA-11 PET findings (SUV ${ }_{\text {max }}$,

Table $3{ }^{68}$ Ga-PSMA-11 PET findings

\begin{tabular}{lc}
\hline Characteristic & Patients \\
\hline miTNM classification, no. (\%), $n=186$ & \\
miT status & $73(39.2 \%)$ \\
$2 \mathrm{u}$ & $52(28 \%)$ \\
$2 \mathrm{~m}$ & $27(14.5 \%)$ \\
$3 \mathrm{a}$ & $24(12.9 \%)$ \\
$3 \mathrm{~b}$ & $10(5.4 \%)$ \\
4 & \\
miN status & $168(90.3 \%)$ \\
0 & $7(3.8 \%)$ \\
1 & $11(5.9 \%)$ \\
2 & \\
miM status & $179(96.2 \%)$ \\
0 & $3(1.6 \%)$ \\
$1 \mathrm{a}$ & $4(2.2 \%)$ \\
$1 \mathrm{~b}$ & $183^{\mathrm{a}}$ \\
PSMA-PET findings of prostatic lesions, median (IQR), $n$ & $10.6(6.4-18.9)$ \\
SUV & \\
SUV & $6.2(3.2-11.0)$ \\
TV & $3.9(1.7-10.5)$ \\
TL & $24.7(15.9-44.4)$ \\
\hline
\end{tabular}

IQR interquartile range, PET positron emission tomography, PSMA prostatespecific membrane antigen, SUV standardized uptake value, $T L$ total lesion, $T V$ tumor volume

${ }^{\text {a }}$ Three patients had PSMA negative prostate cancer. miTNM of these patients were classified based on MRI images
$\left.\mathrm{SUV}_{\text {mean }}, \mathrm{TV}, \mathrm{TL}\right)$ of prostatic lesions were analyzed with 183 patients because three patients were reported negative PSMA prostate cancer. Table 3 lists information from ${ }^{68}$ Ga-PSMA-11 PET. In three cases with PSMA-PET negative primary tumor cross sectional imaging was used to determine the miTNM stage.

Based on the proposed stage group system combining the miTNM staging and $\mathrm{SUV}_{\max }$ of 20 (10.8\%), 96 (51.6\%), 40 (21.5\%), 7 (3.8\%), 16 (8.6\%) and 7 (3.8\%) into the stage groups IA, IB, IIA, IIB, III and IV was performed, respectively.

\section{Correlation of 68Ga-PSMA-11 PET parameters with histopathology}

The sensitivity and specificity of ${ }^{68}$ Ga-PSMA-11 PET detecting pelvic lymph nodes metastasis were $40.6 \%$ and $96.8 \%$ (13/32 and 149/154, respectively). Of $94 \mathrm{pT} \geq 3 \mathrm{a}$ prostatic lesions, $45.7 \%(n=43)$ were detected $(\mathrm{miT} \geq 3 \mathrm{a})$ by ${ }^{68} \mathrm{Ga}-\mathrm{PSMA}-11$ PET. $80.4 \%(n=74)$ of pT2 prostatic lesions were correctly classified as miT2. Cross tables are presented in Additional file 1: Tables S1 and S2.

In the univariate analysis (Additional file 1: Table S3), a significant association was detected between a positive surgical margin and the following parameters: high miT status (miT $\geq 3 \mathrm{a}$, OR: $3.38, p=0.004)$, miN1 status

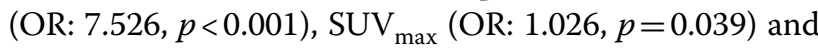
TL (OR: $1.007, p=0.021$ ). In the multivariate analysis (Table 4), miN1 (OR: 5.428, $p=0.004$ ) was significantly associated with a positive surgical margin. Moreover, a significant association was present between $\mathrm{miT} \geq 3 \mathrm{a}$ and GS $\geq 8$ (OR: 2.696, $p=0.003$ ) (Table 5).

\section{Predictors of BCR-free survival}

Kaplan-Meier curves for BCR-free survival with different clinicopathological and miTNM-derived parameters are shown in Figures 1, 2, 3 and Additional file 1: Fig. S2.

Table 4 Multivariate analysis for the association of ${ }^{68} \mathrm{Ga}-\mathrm{PSMA}-11$ PET findings with surgical margin status

\begin{tabular}{|c|c|c|c|c|}
\hline & No. of patients & Odds ratio & $95 \% \mathrm{Cl}$ & $p$ value* \\
\hline \multicolumn{5}{|c|}{ miTNM classification, no., $n=186$} \\
\hline \multicolumn{5}{|l|}{ miT status } \\
\hline 2 & 125 & Reference & & \\
\hline$\geq 3 a$ & 61 & 2.065 & $0.802-5.315$ & 0.133 \\
\hline \multicolumn{5}{|l|}{ miN status } \\
\hline No LN metastasis & 168 & Reference & & \\
\hline With LN metastasis & 18 & 5.428 & $1.708-17.249$ & 0.004 \\
\hline $\mathrm{SUV}_{\max }$ of prostatic lesions & 183 & 1.015 & $0.988-1.044$ & 0.282 \\
\hline TL of prostatic lesions & 183 & 1.004 & $0.998-1.011$ & 0.166 \\
\hline
\end{tabular}

Cl confidence interval, LN lymph node, PET positron emission tomography, PSMA prostate-specific membrane antigen, SUV standardized uptake value, TL total lesion, $T V$ tumor volume

*Significant associations are given in bold 
Table 5 Univariate analysis for the association of ${ }^{68}$ Ga-PSMA-11 PET findings with Gleason Score

\begin{tabular}{|c|c|c|c|c|}
\hline & No. of patients & Odds ratio & $95 \% \mathrm{Cl}$ & $p$ value* \\
\hline \multirow{2}{*}{\multicolumn{5}{|c|}{$\begin{array}{l}\text { miTNM classification, no., } n=186 \\
\text { miT status }\end{array}$}} \\
\hline & & & & \\
\hline 2 & 125 & Reference & & \\
\hline$\geq 3 \mathrm{a}$ & 61 & 2.696 & $1.39-5.23$ & 0.003 \\
\hline \multicolumn{5}{|l|}{ miN status } \\
\hline No LN metastasis & 168 & Reference & & \\
\hline With LN metastasis & 18 & 2.187 & $0.812-5.887$ & 0.122 \\
\hline$S U V_{\text {mean }}$ of prostatic lesions & 183 & 1.020 & $0.986-1.056$ & 0.248 \\
\hline SUV $_{\max }$ of prostatic lesions & 183 & 1.017 & $0.995-1.040$ & 0.138 \\
\hline TV of prostatic lesions & 183 & 0.981 & $0.941-1.022$ & 0.353 \\
\hline TL of prostatic lesions & 183 & 1.006 & $1-1.012$ & 0.056 \\
\hline
\end{tabular}

Cl confidence interval, $L N$ lymph node, PET positron emission tomography, PSMA prostate-specific membrane antigen, SUV standardized uptake value, $T L$ total lesion, TV tumor volume

*Significant associations are given in bold

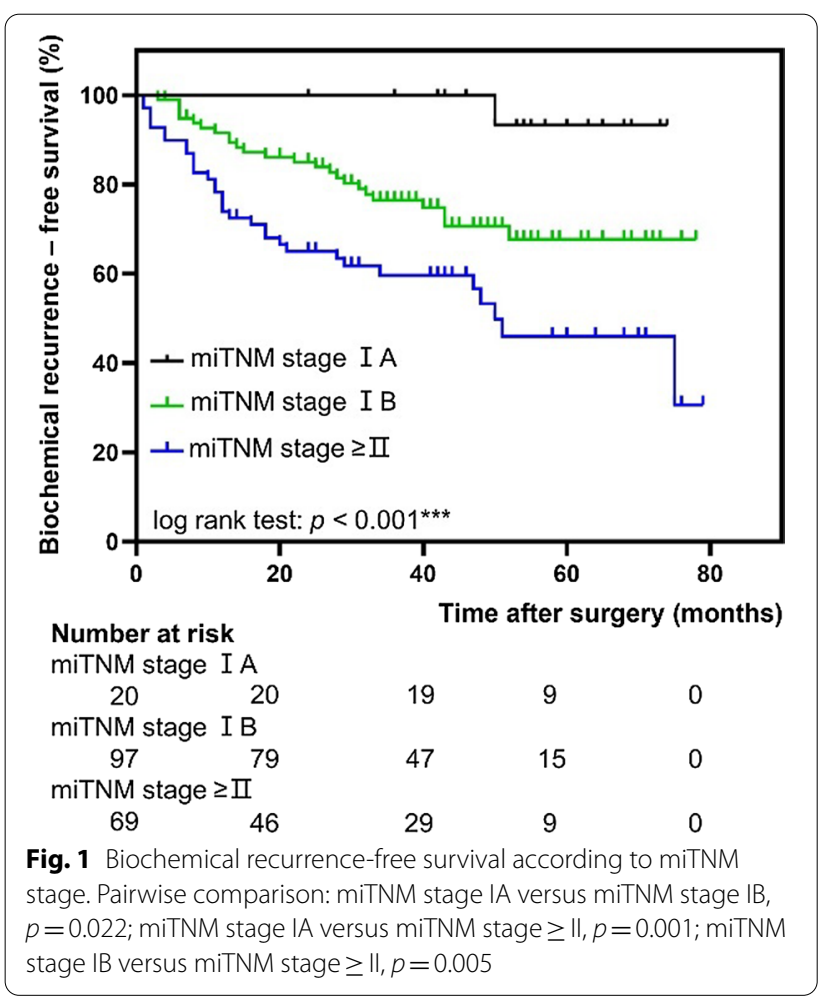

The miTNM derived parameters miT2 versus miT3 disease or higher (log-rank, $p<0.001$, Fig. $2 \mathrm{a}$ ), miN0 versus miN1/2 (log-rank, $p=0.005$, Fig. $2 \mathrm{~b}$ ) and miTNM stage group IA compared with miTNM stage group IB (log-rank, $p=0.022$, Fig. 1) were associated with significantly different BCR-free survival rate. Lower $S_{U} V_{\text {mean }}$ and $\mathrm{SUV}_{\max }$ as quantitative parameters from ${ }^{68} \mathrm{Ga}-$ PSMA-11 PET were also associated with longer BCR-free in patients (Fig. 3c, d, log-rank, $p=0.035, p=0.037$, respectively).

The following pathological features were associated with longer BCR-free survival: pT2 versus $\geq$ pT3a (logrank, $p<0.001$, Additional file 1: Fig. S2A). pN0 versus pN1 (log-rank, $p<0.001$, Additional file 1: Fig. S2B), lower Gleason Grades $(\mathrm{GS}<8)$ (log-rank, $p<0.001$, Additional file 1: Fig. S2C) and negative surgical margins (logrank, $p<0.001$, Additional file 1: Fig. S2D).

Results from a univariate Cox regression analysis investigating preoperative and postoperative risk factors for BCR is presented in Table 6 . We found that following factors were significantly associated with BCR-free survival in prostate cancer patients: clinical data including age (HR: 1.056, 95\% CI 1.018-1.096, $p=0.004$ ) and initial PSA (iPSA) (HR: 1.021, 95\% CI 1.007-1.035, $p=0.003$ ); pathological data including Gleason score $(\mathrm{GS} \geq 8$ vs. GS $<8$, HR: 5.097, 95\% CI 3.013-8.625, $p<0.001)$, pT stage (pT $\geq 3$ vs. $\mathrm{pT}<3$, HR: $2.935,95 \%$ CI $1.665-5.173$, $p<0.001$ ), $\mathrm{pN}$ stage (pN1 vs. pN0, HR: $3.378,95 \% \mathrm{CI}$ $1.901-6, p<0.001$ ) and surgical margin (positive vs. negative, HR: 3.421, 95\% CI 1.890-6.193, $p<0.001)$. Imaging parameters include miT stage $(\mathrm{miT} \geq 3 \mathrm{a}$ vs. $\mathrm{miT}<3$, HR: 2.811, 95\% CI 1.673-4.722, $p<0.001$ ), miN stage (miN1 vs. miN0, HR: $2.691,95 \%$ CI 1.311-5.527, $p=0.007$ ), $\mathrm{SUV}_{\text {mean }}$ of prostatic lesions (HR: 1.019, 95\% CI 1.0021.036, $p=0.028$ ), $\mathrm{SUV}_{\max }$ of prostatic lesions (HR: 1.015, 95\% CI 1.004-1.026, $p=0.008)$ and TV of prostatic lesions (HR: 0.948, 95\% CI 0.909-0.988, $p=0.011$ ).

In the multivariate Cox regression analysis (Table 7), the following factors were independent predictors for BCR-free survival: serum PSA level (HR: 1.024, 95\% CI 1.005-1.043, $p=0.014$ ), advanced pathological Gleason Score (GS $\geq 8$ vs. GS $<8$, HR: 3.253, 95\% CI 1.779-5.950; 

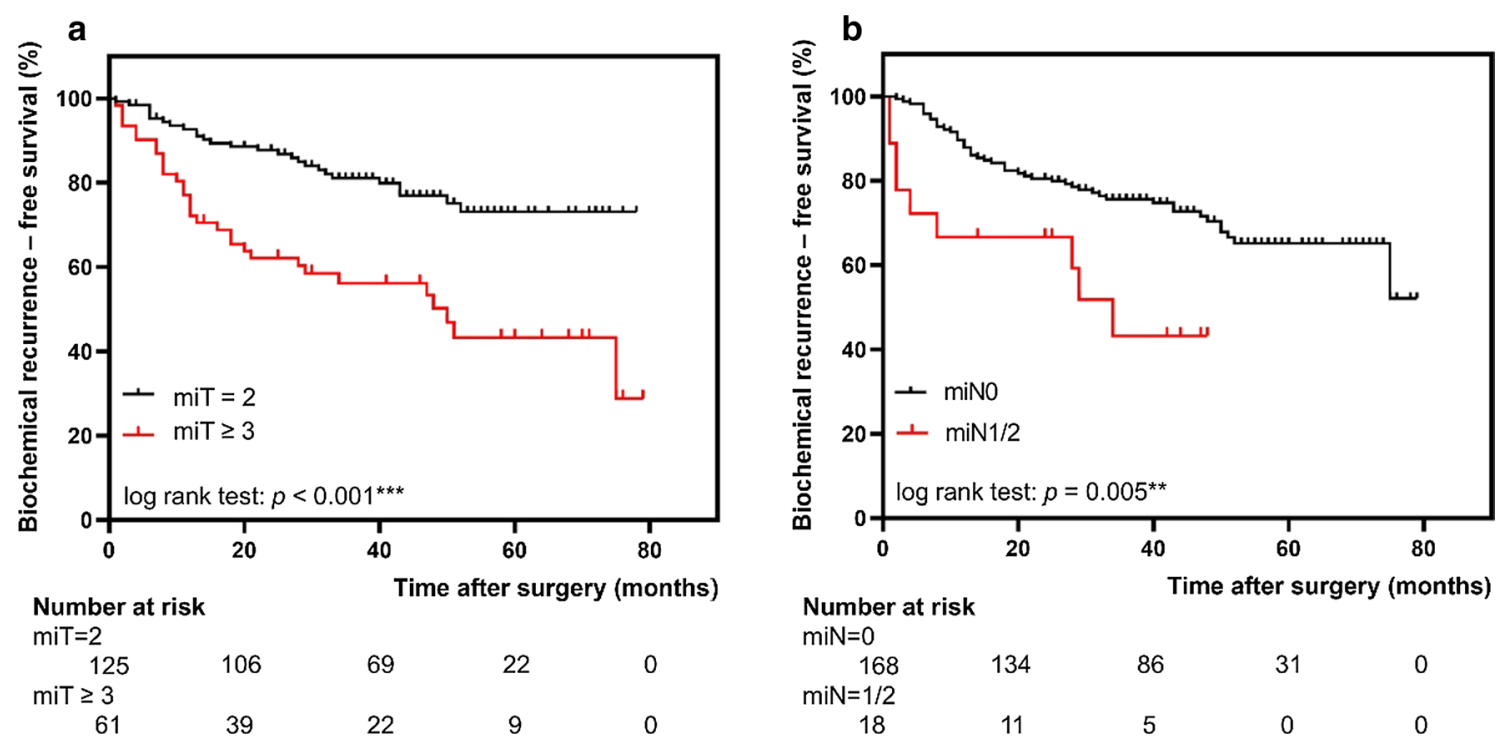

Fig. 2 Longer biochemical recurrence-free survival was associated with $\mathbf{a}$ miT $=2$ and $\mathbf{b}$ miN $=0$

$p<0.001$ ) and miT stage (miT $\geq 3$ a vs. $\mathrm{miT}<3$, HR: 1.941, $95 \%$ CI 1.047-3.599, $p=0.035)$.

\section{Discussion}

Our retrospective analysis demonstrates the potential predictive value of ${ }^{68} \mathrm{Ga}$-PSMA-11 PET findings for BCR-free survival of primary prostate cancer after RP. Information from the recently introduced miTNM classification is associated with outcome. In addition, several correlations between quantitative and qualitative measures from ${ }^{68} \mathrm{Ga}$-PSMA-11 PET and pathological parameters have been observed. Prognostic tools of BCR are required and essential for improving treatment management of prostate cancer patients and reducing prostate cancer-associated mortality of patients developing BCR after primary treatment [32]. With the successful application of PSMA-ligand PET for primary staging in prostate cancer patients, clinical studies are necessary to investigate its predictive value. PSMA-ligand PET is increasingly used for selection, monitoring and individualization of prostate cancer treatments.

The present analysis is the first to investigate the association of miTNM classification from preoperative ${ }^{68}$ Ga-PSMA-11 PET imaging and postoperative histopathological findings and the potential of miTNM reporting to serve as predictors for BCR after RP. Consequently, we performed a prognostic validation of the miTNM system as a framework for PSMA-ligand PET reporting in a relatively large patient cohort. Preoperative ${ }^{68}$ Ga-PSMA-11 PET and miTNM classification could help to stratify risk for BCR after RP and could potentially further influence the clinical patient management.
Previous studies have extensively assessed the predictors of BCR. Clinicopathological characteristics, including pathological aggressive GS [5-9], positive nerve invasion [8], pathological $\mathrm{T}$ stage [7] and preoperative PSA [3-6], were proven to have a strong association with BCR. Our present results are in accordance with literature data. The data from our analysis outline serum PSA level (HR: 1.024, 95\% CI 1.005-1.043, $p=0.014)$ and advanced pathological Gleason score $(\mathrm{GS} \geq 8$ vs. $\mathrm{GS}<8, \mathrm{HR}: 3.253,95 \%$ CI $1.779-5.950$; $p<0.001)$ as important histopathological predictors of BCR.

In addition, and novel compared to the current literature our data indicate that also parameters from ${ }^{68} \mathrm{Ga}$ PSMA-11 PET might serve as non-invasive biomarkers. We identified a miT stage $\geq 3 \mathrm{a}$ in ${ }^{68} \mathrm{Ga}$-PSMA-11 PET as surrogate for higher GS (OR: 2.696, 95\% CI 1.395.23, $p=0.003$ ) and worse BCR-free survival (HR: 1.941, 95\% CI 1.047-3.599, $p=0.035$ ). Notably, pelvic lymph node metastases in ${ }^{68} \mathrm{Ga}$-PSMA-11 PET were not detected as an independent predictor for BCR in this study (HR: 1.233, 95\% CI 0.389-3.908, $p=0.722$ ). However, the BCR-free survival differed significantly between miN0 and miN1 group (log-rank, $p=0.005$ ). Interestingly also Raheem et al. have failed to detect lymph nodes in histopathology as an independent predictor to BCR after RP in a study including 359 patients [4]. Of note, the sample size of miN1 group $(n=18)$ in our cohort was relatively small. Thus, the interpretation of results should be with caution, and further studies including more miN1 patients are needed to clarify the predictive value of miN classification for BCR after RP. 

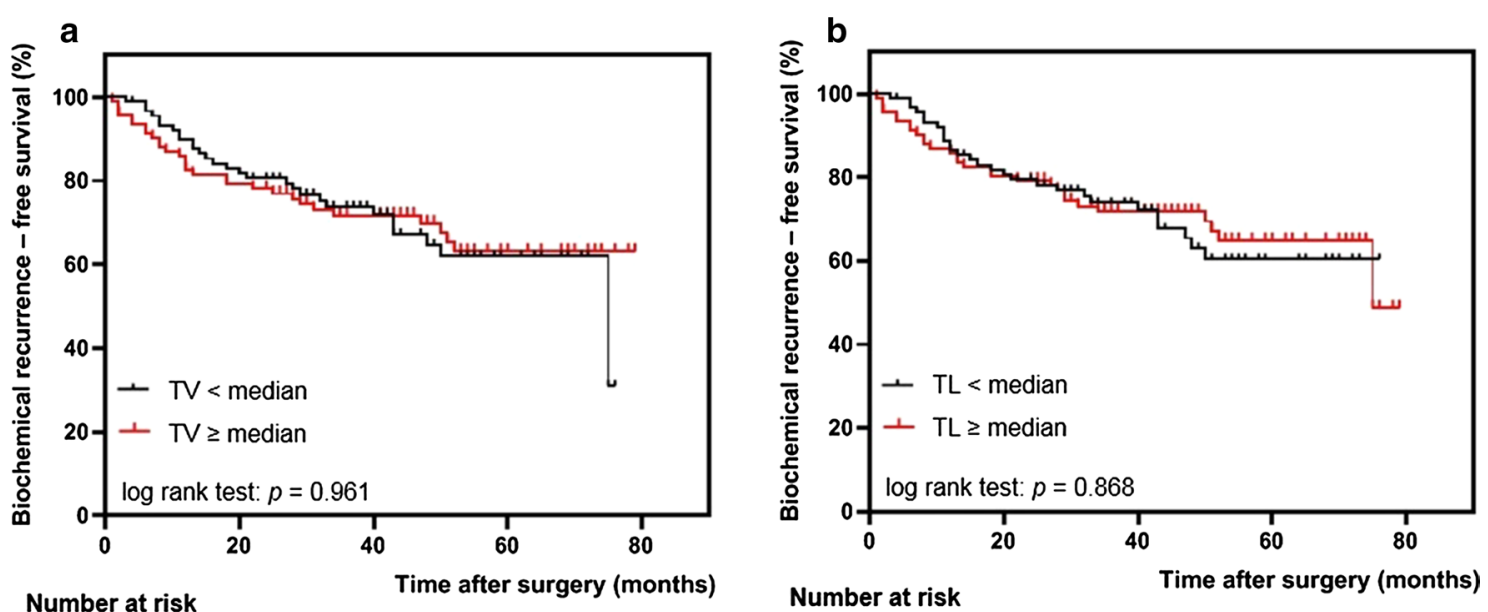

\begin{tabular}{|c|c|c|c|}
\hline 91 & 72 & 39 & 13 \\
\hline $\begin{array}{c}T V \geq \operatorname{median}_{92} \\
\text { ment }\end{array}$ & 73 & 51 & 19 \\
\hline
\end{tabular}

$\begin{array}{ccccc}\mathrm{TL}<\text { median } & & & & \\ 91 & 70 & 41 & 12 & 0 \\ \mathrm{TL} \geq \text { median } & & & & \\ 92 & 75 & 49 & 20 & 0\end{array}$
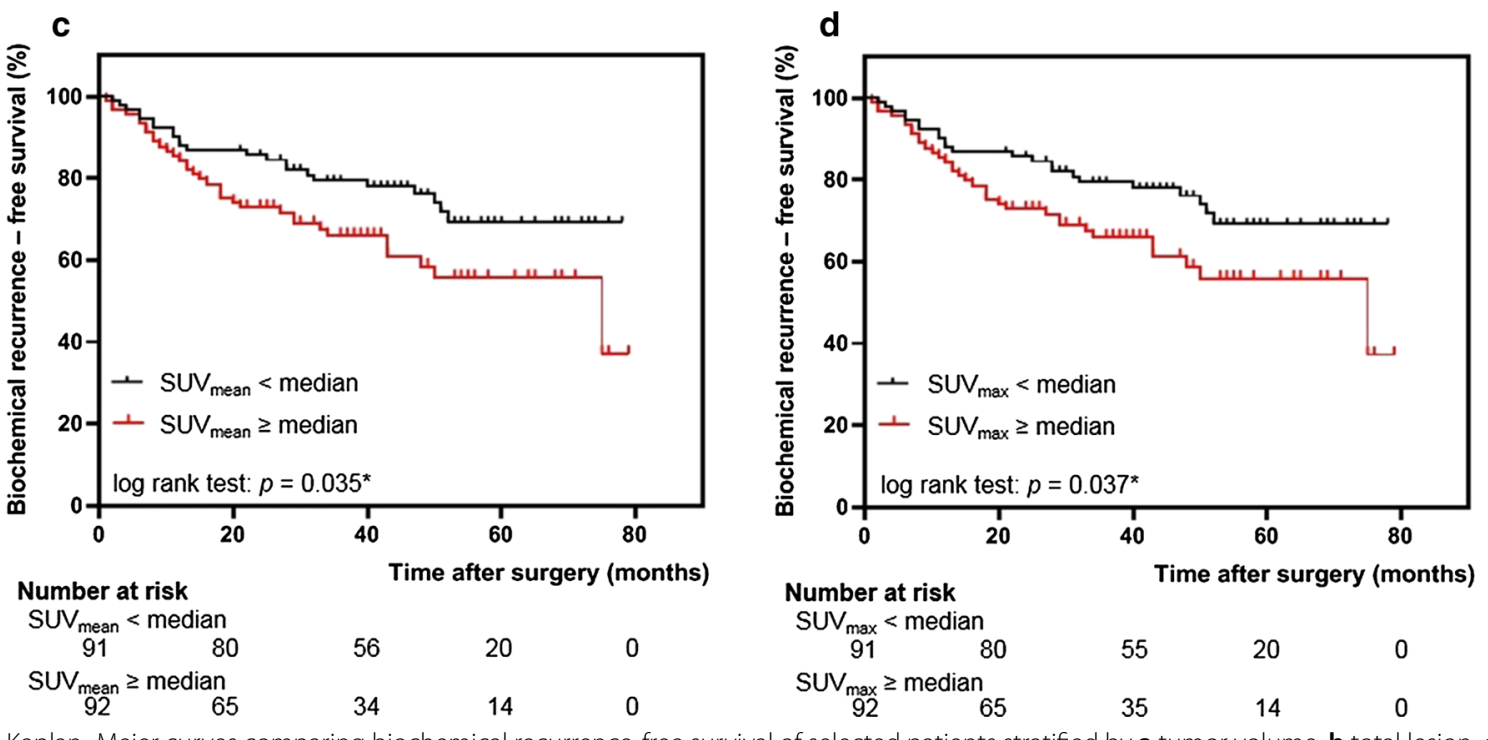

Fig. 3 Kaplan-Meier curves comparing biochemical recurrence-free survival of selected patients stratified by a tumor volume, $\mathbf{b}$ total lesion, $\mathbf{c}$ $S_{S U V}$ mean and $\mathbf{d} S U V_{\text {max }}$ Longer biochemical recurrence-free survival was associated with lower SUV $V_{\text {mean }}$ and SUV $V_{\text {max }}$

Besides, our results indicate a negative association of TV with BCR-free survival (HR: 0.934, 95\% CI 0.883$0.988, p=0.017)$. Contrarily, Choi et al. have reported a significantly higher BCR-free survival rate in pT2 prostate cancer patients with percent tumor volume $\leq 7.5 \%$, which was assessed using histological samples $(p<0.001)$ [33]. This is partly related to the methods of obtaining tumor volume and studies are necessary to assess the standard of TV calculation from ${ }^{68}$ Ga-PSMA-11 PET and pathological samples.

With this work we also introduced a stage grading system based on the recent proposed molecular staging system (miTNM staging system, version 1.0) combined with quantitative parameters. It is intended to mirror the AJCC staging system based on clinicopathological parameters which has proven to be a fundamental tool that also informs treatment decisions [34]. Bhindi et al. have confirmed the ability of the $8^{\text {th }}$ edition to predict oncologic outcomes [35]. However, the AJCC staging system utilized clinical or pathological TNM stage and no parameters from imaging. With the increasing use of PSMA-ligand PET in clinical routine, a logical next step is to use information from non-invasive imaging prior to definite treatment for risk stratification.

We have shown that the miT stage is an independent predictor of $\mathrm{BCR}$, and we observed a widely 
Table 6 Univariable analysis for the association of baseline factors with BCR-free survival

\begin{tabular}{|c|c|c|c|c|}
\hline & No. of patients & Hazard ratio & $95 \% \mathrm{Cl}$ & $p$ value* \\
\hline \multicolumn{5}{|l|}{ Clinical data } \\
\hline Age & 186 & 1.056 & $1.018-1.096$ & 0.004 \\
\hline ¡PSA & 184 & 1.021 & $1.007-1.035$ & 0.003 \\
\hline \multicolumn{5}{|l|}{ Pathological data } \\
\hline \multicolumn{5}{|c|}{ Gleason score in surgical specimen, no., $n=186$} \\
\hline $6-7$ & 133 & Reference & & \\
\hline $8-10$ & 53 & 5.097 & $3.013-8.625$ & $<0.001$ \\
\hline \multicolumn{5}{|l|}{ Pathological stage, no., $n=186$} \\
\hline \multicolumn{5}{|l|}{ pT status } \\
\hline 2 & 92 & Reference & & \\
\hline$\geq 3$ & 94 & 2.935 & $1.665-5.173$ & $<0.001$ \\
\hline \multicolumn{5}{|l|}{ pN status } \\
\hline 0 & 154 & Reference & & \\
\hline 1 & 32 & 3.378 & $1.901-6.000$ & $<0.001$ \\
\hline \multicolumn{5}{|l|}{ Surgical margin, no., $n=180$} \\
\hline Negative & 152 & Reference & & \\
\hline Positive & 28 & 3.421 & $1.890-6.193$ & $<0.001$ \\
\hline \multicolumn{5}{|l|}{ Imaging parameters } \\
\hline \multicolumn{5}{|l|}{ miTNM classification, no., $n=186$} \\
\hline \multicolumn{5}{|l|}{ miT status } \\
\hline 2 & 125 & Reference & & \\
\hline$\geq 3 \mathrm{a}$ & 61 & 2.811 & $1.673-4.722$ & $<0.001$ \\
\hline \multicolumn{5}{|l|}{ miN status } \\
\hline No LN metastasis & 168 & Reference & & \\
\hline With LN metastasis & 18 & 2.691 & $1.311-5.527$ & 0.007 \\
\hline$S U V_{\text {mean }}$ of prostatic lesions & 183 & 1.019 & $1.002-1.036$ & 0.028 \\
\hline \multicolumn{5}{|c|}{$\mathrm{SUV}_{\text {mean }}$ of prostatic lesions, no. $n=183$} \\
\hline$<$ median & 91 & Reference & & \\
\hline$\geq$ median & 92 & 1.752 & $1.030-2.981$ & 0.039 \\
\hline SUV $V_{\max }$ of prostatic lesions & 183 & 1.015 & $1.004-1.026$ & 0.008 \\
\hline \multicolumn{5}{|c|}{ SUV $_{\max }$ of prostatic lesions, no. $n=183$} \\
\hline$<$ median & 91 & Reference & & \\
\hline$\geq$ median & 92 & 1.744 & $1.025-2.968$ & 0.040 \\
\hline TV of prostatic lesions & 183 & 0.948 & $0.909-0.988$ & 0.011 \\
\hline \multicolumn{5}{|l|}{ TV of prostatic lesions, no., $n=183$} \\
\hline$<$ median & 91 & Reference & & \\
\hline$\geq$ median & 92 & 0.987 & $0.587-1.661$ & 0.962 \\
\hline TL of prostatic lesions & 183 & 1.003 & $1.000-1.006$ & 0.072 \\
\hline \multicolumn{5}{|l|}{ TL of prostatic lesions, no., $n=183$} \\
\hline$<$ median & 91 & Reference & & \\
\hline$\geq$ median & 92 & 0.957 & $0.568-1.612$ & 0.869 \\
\hline
\end{tabular}

$B C R$ biochemical recurrence, $C l$ confidence interval, $i P S A$ initial PSA, IQR interquartile range, $L N$ lymph node, $P E T$ positron emission tomography, $P S A$ prostate-specific antigen, PSMA prostate-specific membrane antigen, SUV standardized uptake value, $T L$ total lesion, $T V$ tumor volume

*Significant associations are given in bold

varying prognosis in the miT2 stage patients. Similarly, a recent study has revealed that high intraprostatic ${ }^{68} \mathrm{Ga}$ PSMA-11 uptake $\left(\mathrm{SUV}_{\max }>8\right)$ predicts short progression-free survival rate among patients with GS $3+4$ on biopsy [36]. The significant difference in BCR-free survival rate has been confirmed in IA and IB stage groups. Our findings propose that a SUV $\max$ cut-off extracted from literature could further stratify the group of miT2 
Table 7 Multivariable analysis for the association of baseline factors with BCR-free survival

\begin{tabular}{|c|c|c|c|c|}
\hline & No. of patients & Hazard ratio & $95 \% \mathrm{Cl}$ & $p$ value* \\
\hline \multicolumn{5}{|l|}{ Clinical data } \\
\hline \multicolumn{5}{|l|}{ Age, no., $n=186$} \\
\hline Continuous & & 1.030 & $0.991-1.071$ & 0.133 \\
\hline \multicolumn{5}{|l|}{ iPSA, no., $n=184$} \\
\hline Continuous & & 1.024 & $1.005-1.043$ & 0.014 \\
\hline \multicolumn{5}{|l|}{ Pathological data } \\
\hline \multicolumn{5}{|c|}{ Gleason score in surgical specimen, no., $n=186$} \\
\hline $6-7$ & 133 & Reference & & \\
\hline $8-10$ & 53 & 3.253 & $1.779-5.950$ & $<0.001$ \\
\hline \multicolumn{5}{|l|}{ pT status, no., $n=186$} \\
\hline 2 & 92 & Reference & & \\
\hline 3 & 94 & 1.471 & $0.773-2.797$ & 0.239 \\
\hline \multicolumn{5}{|l|}{ pN status, no., $n=186$} \\
\hline No LN metastasis & 154 & Reference & & \\
\hline With LN metastasis & 32 & 1.027 & $0.418-2.525$ & 0.954 \\
\hline \multicolumn{5}{|c|}{ Surgical margin, no., $n=180$} \\
\hline Negative & 152 & Reference & & \\
\hline Positive & 28 & 1.539 & $0.716-3.305$ & 0.269 \\
\hline \multicolumn{5}{|l|}{ Imaging parameters } \\
\hline \multicolumn{5}{|c|}{ miT status from PSMA PET, no., $n=186$} \\
\hline 2 & 125 & Reference & & \\
\hline$\geq 3 a$ & 61 & 1.941 & $1.047-3.599$ & 0.035 \\
\hline \multicolumn{5}{|c|}{ miN status from PSMA PET, no., $n=186$} \\
\hline No LN metastasis & 168 & Reference & & \\
\hline With LN metastasis & 18 & 1.233 & $0.389-3.908$ & 0.722 \\
\hline \multicolumn{5}{|l|}{ SUV mean $_{1}$ no., $n=183$} \\
\hline Continuous & & 0.743 & $0.491-1.123$ & 0.159 \\
\hline \multicolumn{5}{|l|}{ SUV $\max _{\text {mo., }} n=183$} \\
\hline Continuous & & 1.202 & $0.943-1.532$ & 0.137 \\
\hline \multicolumn{5}{|l|}{ TV } \\
\hline Continuous & & 0.934 & $0.883-0.988$ & 0.017 \\
\hline
\end{tabular}

$B C R$ biochemical recurrence, $C l$ confidence interval, iPSA initial PSA, LN lymph node, PET positron emission tomography, PSA prostate-specific antigen, PSMA prostatespecific membrane antigen, SUV standardized uptake value, $T V$ tumor volume

*Significant associations are given in bold

primary disease into patients with more aggressive disease and worse prognosis. Further studies are necessary for prognostic validation of other stage groups.

The present study has several limitations. It is a retrospective analysis and includes only patients from a single center, which can introduce potential bias. Despite inclusions of a large number of patients, the sample size of patients in the miM1 group was too small to conduct meaningful analysis. This is mainly related to the fact that most patients with extrapelvic metastases do not undergo primary curative RP but either get systemic treatment with or without local treatment. This explains the low number of patients in miTNM stage group III and IV. In summary, further prospective investigations with large patient numbers are necessary to fully investigate the potential of the miTNM staging and our proposed grading system to predict patient outcome after curative intent RP.

\section{Conclusion}

Our retrospective analysis indicates that the miTNM framework developed to standardize PSMA-ligand PET reported is independently associated with BCRfree survival of primary prostate cancer after RP. We demonstrated significant associations between ${ }^{68} \mathrm{Ga}$ PSMA-11 PET findings and histopathological parameters. In summary, our results outline that the miTNM classification and the presented further development of a 
miTNM-based stage group system can serve as non-invasive imaging biomarkers of risk stratification for primary prostate cancer patients. However, further and prospective studies including patients with different treatments and stages are needed to fully assess the predictive value of PSMA-ligand PET imaging in the setting of newly diagnosed prostate cancer.

\begin{abstract}
Abbreviations
${ }^{68} \mathrm{Ga}$ : Gallium-68; AJCC: American Joint Committee on Cancer; BCR: Biochemical recurrence; BSI: Bone scan index; ${ }^{11} \mathrm{C}$ : Carbon-11; Cl: Confidence interval; CT: Computed tomography; DNA: Deoxyribonucleic acid; ESCC: Esophageal squamous cell carcinoma; FDG: Fluorodeoxyglucose; GS: Gleason score; HR: Hazard ratio; iPSA: Initial PSA; IQR: Interquartile range; mCRPC: Metastatic castration-resistant prostate cancer; miTNM: Imaging TNM classification; MRI: Magnetic resonance imaging; OR: Odds ratio; PET: Positron emission tomography; PSA: Prostate-specific antigen; PSMA: Prostate-specific membrane antigen; RP: Radical prostatectomy; SD: Standard deviation; SUV: Standardized uptake value; TL: Total lesion; TV:Tumor volume; VOI: Volume of interest.
\end{abstract}

\section{Supplementary Information}

The online version contains supplementary material available at https://doi. org/10.1186/s13550-021-00818-2.

Additional file 1. Supplementary files. Supplementary table 1. Distribution of pT and miT. Supplementary table 2. Distribution of $\mathrm{pN}$ and miN. Supplementary table 3. Univariate analysis for the association of ${ }^{68} \mathrm{Ga}-\mathrm{PSMA}-11$ PET findings with surgical margin status. Supplementary Fig. 1. Flowchart of inclusion and exclusion steps. Supplementary Fig. 2. Longer biochemical recurrence-free survival was associated with (A) $\mathrm{pT}=2$, (B) $\mathrm{pN}=0$, (C) Gleason Score $<8$ and (D) negative surgical margin.

\section{Acknowledgements}

Not applicable.

\section{Authors' contributions}

HW was involved in study design, data acquisition, methodology and manuscript writing. TA was involved in data acquisition. CW supported statistical analysis. TL, KS, IR, TH, TM, KK, HJW, and WW supported the data collection and revised the manuscript. ME was involved in study design, data acquisition and critical manuscript revising. All authors read and approved the final manuscript.

\section{Funding}

Open Access funding enabled and organized by Projekt DEAL. Matthias Eiber, Hans-Juergen Wester and Katja Steiger received funding from the SFB 824 (DFG Sonderforschungsbereich 824, Project B11 and Z2) from the Deutsche Forschungsgemeinschaft, Bonn, Germany. Hui Wang received a scholarship from the China Scholarship Council (CSC).

\section{Availability of data and materials}

The datasets generated during and/or analyzed during the current study are available from the corresponding author on a reasonable request.

\section{Declarations}

\section{Ethics approval and informed consent}

All procedures performed in studies involving human participants were in accordance with the Declaration of Helsinki. This retrospective study has been approved by the Ethics Committee of the Technical University Munich (750/20 $\mathrm{S}-\mathrm{KH})$.

\section{Consent for publication}

Not applicable.

\section{Competing interests}

ME reports patent application for rhPSMA and prior consulting activities for Blue Earth Diagnostics, Progenics Pharmaceuticals, Point Biopharma and Janssen Pharmaceuticals. Other authors declare that they have no competing interests.

\section{Author details}

${ }^{1}$ Department of Nuclear Medicine, Klinikum rechts der Isar, Technical University Munich, Ismaninger Str. 22, 81675 Munich, Germany. ${ }^{2}$ Department of Urology, Klinikum rechts der Isar, Technical University Munich, Ismaninger Str. 22, 81675 Munich, Germany. ${ }^{3}$ Martini-Klinik Prostate Cancer Center, University Hospital Hamburg-Eppendorf, Martinistr. 52, 20246 Hamburg, Germany. ${ }^{4}$ Institute of Pathology, School of Medicine, Technical University Munich, Trogerstr. 18, 81675 Munich, Germany. ${ }^{5}$ Department of Urology, University Hospital Hamburg-Eppendorf, Martinistr. 52, 20246 Hamburg, Germany. ${ }^{6}$ Pharmaceutical Radiochemistry, Technical University of Munich, Walther-Meißner-Str. 3, 85748 Garching, Germany.

Received: 7 June 2021 Accepted: 28 July 2021

Published online: 21 August 2021

\section{References}

1. Schwarzenboeck SM, Rauscher I, Bluemel C, Fendler WP, Rowe SP, Pomper MG, et al. PSMA ligands for PET imaging of prostate cancer. J Nucl Med. 2017;58(10):1545-52.

2. Paller CJ, Antonarakis ES, Eisenberger MA, Carducci MA. Management of patients with biochemical recurrence after local therapy for prostate cancer. Hematol Oncol Clin North Am. 2013;27(6):1205-19, viii.

3. Qi P, Tsivian M, Abern MR, Bañez LL, Tang P, Moul JW, et al. Long-term oncological outcomes of men undergoing radical prostatectomy with preoperative prostate-specific antigen $<2.5 \mathrm{ng} / \mathrm{ml}$ and $2.5-4 \mathrm{ng} / \mathrm{ml}$. Urol Oncol. 2013;31(8):1527-32.

4. Abdel Raheem A, Chang KD, Alenzi MJ, Ham WS, Han WK, Choi YD, et al. Predictors of biochemical recurrence after Retzius-sparing robot-assisted radical prostatectomy: Analysis of 359 cases with a median follow-up period of 26 months. Int J Urol. 2018;25(12):1006-14.

5. Freedland SJ, Presti JC, Amling CL, Kane CJ, Aronson WJ, Dorey F, et al. Time trends in biochemical recurrence after radical prostatectomy: results of the SEARCH database. Urology. 2003;61(4):736-41.

6. Aoun F, Albisinni S, Henriet B, Tombal B, Van Velthoven R, Roumeguère T. Predictive factors associated with biochemical recurrence following radical prostatectomy for pathological T2 prostate cancer with negative surgical margins. Scand J Urol. 2017;51(1):20-6.

7. Inagaki T, Kohjimoto Y, Nishizawa S, Kuramoto T, Nanpo Y, Fujii R, et al. PSA at postoperative three months can predict biochemical recurrence in patients with pathological T3 prostate cancer following radical prostatectomy. Int J Urol. 2009;16(12):941-6.

8. Xu X, Li Q, Chang C, Wang X, Xie L. Metabolic syndrome is not associated with prostate cancer recurrence: a retrospective analysis of a chinese cohort. Front Oncol. 2020;10:63.

9. Cao D, Kibel AS, Gao F, Tao Y, Humphrey PA. The Gleason score of tumor at the margin in radical prostatectomy is predictive of biochemical recurrence. Am J Surg Pathol. 2010;34(7):994-1001.

10. Maurer T, Gschwend JE, Rauscher I, Souvatzoglou M, Haller B, Weirich G, et al. Diagnostic efficacy of ${ }^{68}$ gallium-PSMA positron emission tomography compared to conventional imaging for lymph node staging of 130 consecutive patients with intermediate to high risk prostate cancer. J Urol. 2016;195(5):1436-43.

11. Fendler WP, Calais J, Eiber M, Flavell RR, Mishoe A, Feng FY, et al. Assessment of ${ }^{68} \mathrm{Ga}-\mathrm{PSMA}-11$ PET accuracy in localizing recurrent prostate cancer: a prospective single-arm clinical trial. JAMA Oncol. 2019;5(6):856-63.

12. Hofman MS, Lawrentschuk N, Francis RJ, Tang C, Vela I, Thomas P, et al. Prostate-specific membrane antigen PET-CT in patients with highrisk prostate cancer before curative-intent surgery or radiotherapy (proPSMA): a prospective, randomised, multicentre study. Lancet. 2020;395(10231):1208-16.

13. Silver DA, Pellicer I, Fair WR, Heston WD, Cordon-Cardo C. Prostate-specific membrane antigen expression in normal and malignant human tissues. Clin Cancer Res. 1997;3(1):81-5. 
14. Cimadamore A, Cheng M, Santoni M, Lopez-Beltran A, Battelli N, Massar F, et al. New prostate cancer targets for diagnosis, imaging, and therapy: focus on prostate-specific membrane antigen. Front Oncol. 2018;8:653.

15. Mannweiler S, Amersdorfer P, Trajanoski S, Terrett JA, King DGM. Heterogeneity of prostate-specific membrane antigen (PSMA) expression in prostate carcinoma with distant metastasis. Pathol Oncol Res. 2009;15(2):167-72.

16. Minner S, Wittmer C, Graefen M, Salomon G, Steuber T, Haese A, et al. High level PSMA expression is associated with early PSA recurrence in surgically treated prostate cancer. Prostate. 2011;71(3):281-8.

17. Ross JS, Sheehan CE, Fisher HA, Kaufman RP Jr, Kaur P, Gray K, et al. Correlation of primary tumor prostate-specific membrane antigen expression with disease recurrence in prostate cancer. Clin Cancer Res. 2003;9(17):6357-62.

18. Paschalis A, Sheehan B, Riisnaes R, Rodrigues DN, Gurel B, Bertan C, et al. Prostate-specific membrane antigen heterogeneity and DNA repair defects in prostate cancer. Eur Urol. 2019;76(4):469-78.

19. Wieder HA, Brücher BL, Zimmermann F, Becker K, Lordick F, Beer A, et al Time course of tumor metabolic activity during chemoradiotherapy of esophageal squamous cell carcinoma and response to treatment. J Clin Oncol. 2004;22(5):900-8.

20. Giovacchini G, Picchio M, Garcia-Parra R, Briganti A, Abdollah F, Gianolli $\mathrm{L}$, et al. ${ }^{11} \mathrm{C}$-choline PET/CT predicts prostate cancer-specific survival in patients with biochemical failure during androgen-deprivation therapy. J Nucl Med. 2014;55(2):233-41.

21. Armstrong AJ, Kaboteh R, Carducci MA, Damber JE, Stadler WM, Hansen $\mathrm{M}$, et al. Assessment of the bone scan index in a randomized placebocontrolled trial of tasquinimod in men with metastatic castration-resistant prostate cancer (mCRPC). Urol Oncol. 2014;32(8):1308-16.

22. Gafita A, Bieth $M$, Krönke $M$, Tetteh $G$, Navarro F, Wang $H$, et al. qPSMA: semiautomatic software for whole-body tumor burden assessment in prostate cancer using ${ }^{68} \mathrm{Ga}$-PSMA11 PET/CT. J Nucl Med. 2019;60(9):1277-83.

23. Gafita A, Calais J, Hui W, Weber M, Rathke H, Esfandiari R, et al. Predictive factors and prediction nomograms for LuPSMA radioligand therapy in patients with metastatic castration-resistant prostate cancer: an international multicentre retrospective study. J Nucl Med. 2020;61(supplement 1):593-593.

24. Eiber M, Herrmann K, Calais J, Hadaschik B, Giesel FL, Hartenbach M, et al. Prostate Cancer Molecular Imaging Standardized Evaluation (PROMISE): Proposed miTNM Classification for the Interpretation of PSMA-Ligand PET/CT. J Nucl Med. 2018:59(3):469-78.

25. Eder M, Schäfer M, Bauder-Wüst U, Hull WE, Wängler C, Mier W, et al. ${ }^{68} \mathrm{Ga}$ complex lipophilicity and the targeting property of a urea-based PSMA inhibitor for PET imaging. Bioconjug Chem. 2012;23(4):688-97.
26. Martin R, Jüttler S, Müller M, Wester H-J. Cationic eluate pretreatment for automated synthesis of $\left[{ }^{68} \mathrm{Ga}\right]$ CPCR4.2. Nucl Med Biol. 2014;41(1):84-9.

27. Souvatzoglou M, Eiber M, Martinez-Moeller A, Fürst S, Holzapfel K, Maurer T, et al. PET/MR in prostate cancer: technical aspects and potential diagnostic value. Eur J Nucl Med Mol Imaging. 2013;40(Suppl 1):S79-88.

28. Eiber M, Maurer T, Souvatzoglou M, Beer AJ, Ruffani A, Haller B, et al. Evaluation of hybrid ${ }^{68} \mathrm{Ga}$-PSMA ligand PET/CT in 248 patients with biochemical recurrence after radical prostatectomy. J Nucl Med. 2015;56(5):668-74.

29. Hofman MS, Hicks RJ, Maurer T, Eiber M. Prostate-specific membrane antigen PET: clinical utility in prostate cancer, normal patterns, pearls, and pitfalls. Radiographics. 2018;38(1):200-17.

30. Edge SB, Byrd DR, Carducci MA, Compton CC, Fritz A, Greene F. AJCC cancer staging manual. 7th ed. New York: Springer; 2010.

31. Hoffmann MA, Miederer M, Wieler HJ, Ruf C, Jakobs FM, Schreckenberger M. Diagnostic performance of ${ }^{68} \mathrm{Gallium}-\mathrm{PSMA}-11 \mathrm{PET} / \mathrm{CT}$ to detect significant prostate cancer and comparison with ${ }^{18} \mathrm{FEC} \mathrm{PET/CT.} \mathrm{Oncotarget.}$ 2017;8(67):111073-83.

32. Brockman JA, Alanee S, Vickers AJ, Scardino PT, Wood DP, Kibel AS, et al. Nomogram predicting prostate cancer-specific mortality for men with biochemical recurrence after radical prostatectomy. Eur Urol. 2015;67(6):1160-7.

33. Choi SY, Chi BH, Lim B, Kyung YS, You D, Jeong IG, et al. Percent tumor volume vs American Joint Committee on Cancer staging system subclassification for predicting biochemical recurrence in patients with pathologic T2 prostate cancer. J Cancer Res Clin Oncol. 2020;146(2):537-43.

34. Buyyounouski MK, Choyke PL, McKenney JK, Sartor O, Sandler HM, Amin $\mathrm{MB}$, et al. Prostate cancer-major changes in the American Joint Committee on Cancer eighth edition cancer staging manual. CA Cancer J Clin. 2017:67(3):245-53.

35. Bhindi B, Karnes RJ, Rangel LJ, Mason RJ, Gettman MT, Frank I, et al. Independent validation of the American Joint Committee on Cancer 8th edition prostate cancer staging classification. J Urol. 2017;198(6):1286-94.

36. Roberts MJ, Morton A, Donato P, Kyle S, Pattison DA, Thomas P, et al. ${ }^{68} \mathrm{Ga}$-PSMA PET/CT tumour intensity pre-operatively predicts adverse pathological outcomes and progression-free survival in localised prostate cancer. Eur J Nucl Med Mol Imaging. 2021;48(2):477-82.

\section{Publisher's Note}

Springer Nature remains neutral with regard to jurisdictional claims in published maps and institutional affiliations.

\section{Submit your manuscript to a SpringerOpen ${ }^{\circ}$ journal and benefit from:}

- Convenient online submission

- Rigorous peer review

- Open access: articles freely available online

- High visibility within the field

Retaining the copyright to your article

Submit your next manuscript at springeropen.com 